\title{
Dokumentationsprobleme der Schulgeographie am Beispiel der Randstadt Holland
}

Im Westen der Niederlande befinden sich zahlreiche Bevölkerungsschwerpunkte, darunter die Landeshauptstadt Amsterdam (957'731 Einwohner; alle Bevölkerungszahlen beziehen sich auf die Agglomerationen am 1.1.1979), den Regierungssitz Den Haag (671'481 Einwohner), die Hafenstadt Rotterdam (1'014'808 Einwohner) und die Universitätsstadt Utrecht (467'392 Einwohner). Die einzelnen Agglomerationen wachsen aufeinander zu, während sich die dazwischen liegenden Dörfer und Städte rasch entwickeln. Dieses hufeisenförmige Ballungsgebiet nennt man die Randstadt Holland (niederländisch Randstad Holland). Die einzelnen Agglomerationen bestehen einstweilen noch als abgrenzbare Siedlungsflächen (Abb. 1), die aber zusammenzuwachsen drohen. Die Planung von Pufferzonen stellt eine Massnahme gegen die Entstehung einer durchgehenden Bandstadt dar. Weltstadtfunktionen verteilen sich auf die ganze Städtereihe, die einen Bestandteil der westeuropäischen Ballungsgebiete bilden.

Inmitten der Randstadt befindet sich ein landwirtschaftliches Gebiet - zum grossen Teil ältere Polder - , das als das "Grüne Herz" bezeichnet wird (Abb.3).

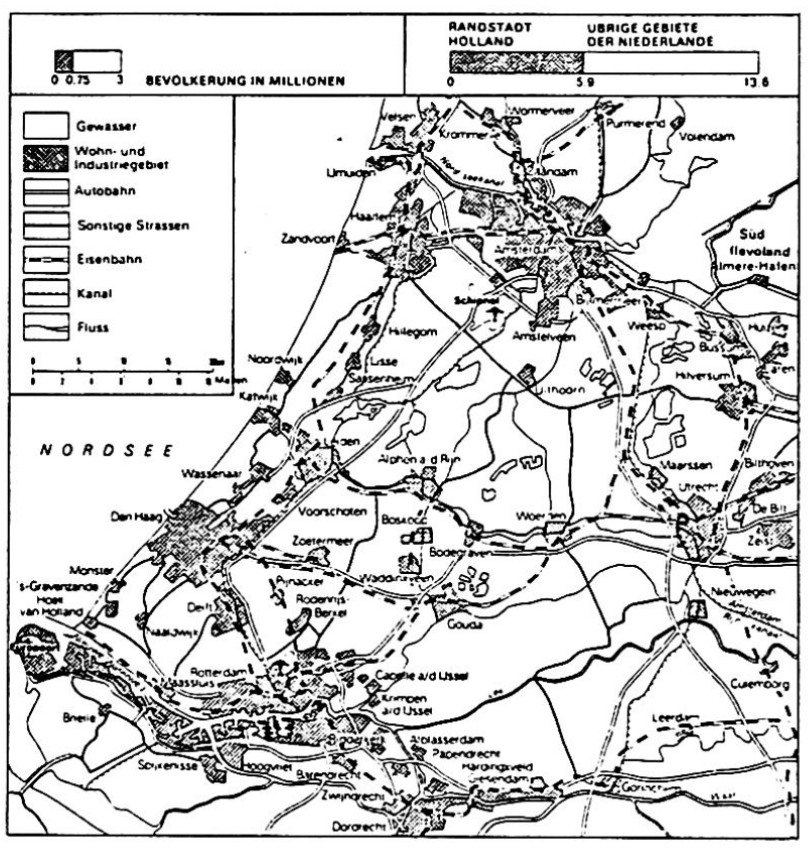

Abb.1: Die Randstadt umzingelt das Grüne Herz: das hufeisenförmige Ballungsgebiet droht ein landwirtschaftliches Kernland zu ersticken.

\section{Probleme der Randstadt}

Während sich die Städte der Niederlande allmählich leeren, wächst die Bevölkerung der gesamten Randstadt einschliesslich des grünen Herzens weiter an. Die Raumplanung stellt sich zur Aufgabe, das Grüne Herz als ländliche Region zu erhalten. Die Siedlungen dieser landwirtschaftlichen Zone sollten wenn möglich nicht mehr wachsen, doch ist die Entwicklung von Orten in einem Netz infrastruktureller Verbindungen schwer zu bremsen (zum Beispiel Gouda 58'560 Einwohner; Alphen 50'350 Einwohner; Woerden 23'400 Einwohner). Ein derartiges landwirtschaftliches Kerngebiet darf nicht der Zersiedlung anheimfallen.

Es ist recht schwierig, der Bevölkerung der Randstadt Holland geeignete Erholungsräume zur Verfügung zu stellen. Das Grüne Herz als landwirtschaftlich intensiv genutztes Polderland kormt mit Ausnahme der Seen - als Erholungsgebiet kaum in Frage. Neu geschaffene Polder im Norden der Randstadt (Südflevoland und das vielleicht später noch trockenzulegende Markerwaard) mitsamt den umliegenden Gewässern könnten diese Aufgabe zumindest für die nördlichen Agglomerationen übernehmen.

Auf alle Fälle ist in Bezug auf die Landnutzung das Grïne Herz ein Konfliktgebiet, während die Randstadt viele städtebauliche, soziale und wirtschaftliche Probleme zu lösen hat. Im Rahmen der niederländischen Raumplanung werden diese Aufgaben systematisch an die Hand genommen.

\section{Die Randstadt Holland als Schulthema}

Für die Niederländer ist die Randstadt ein bekannter Begriff, ein fester Bestandteil ihres täglichen Lebens, ein bedeutendes Kapitel der Geographie ihrer Heimat.

Eine Untersuchung widmete sich der Frage, wieweit die Randstadt Holland im ausländischen Geographieunterricht über die Niederlande Eingang gefunden hat (MEIJER + VERDUIN-MULIER 1979). In 17 von 58 untersuchten ausländischen Schulbüchern findet man im Kapitel "Niederlande" keinen Hinweis auf die Randstadt und ihre Probleme. In zahlreichen Unterrichtswerken und Atlanten wurden zudem viele Fehler, Irrtümer und Verwechslungen aufgedeckt.

François Jeanneret, Dr.phil.nat., Les Vernes 4, 2534 Orvin 


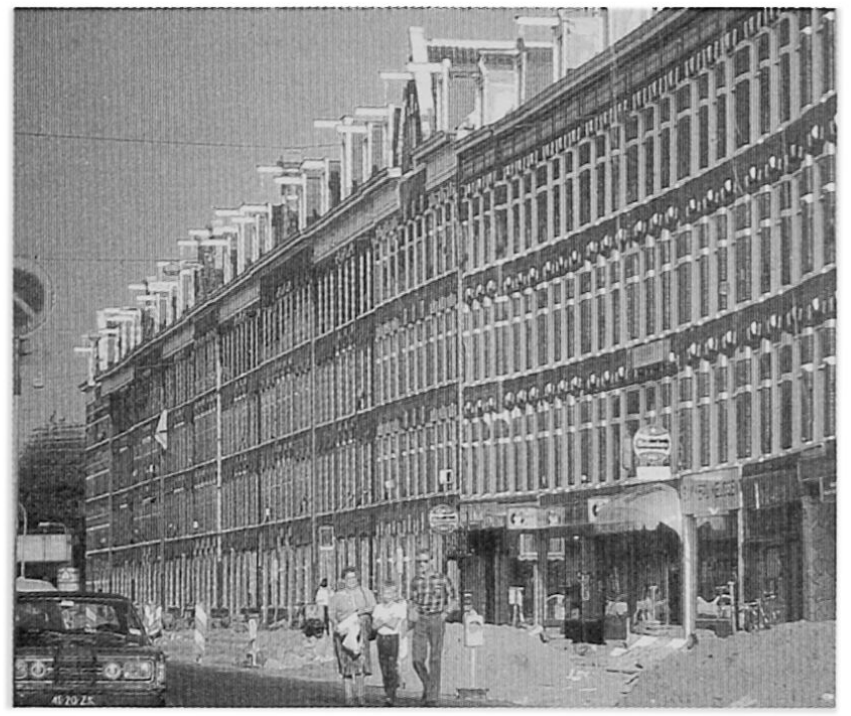

Abb.2: Entvölkerungsprobleme der Industrienationen: der Stadtbewohner meidet immer mehr die Kerngemeinden grosser Agglomerationen der Randstadt, deren Lebensqualität den heutigen Anforderungen nicht mehr genügt (Amsterdam, 93.Quartier).

Wenn auch die Schulbücher nicht schlechthin auf den Unterricht in den Herkunftsländern schliessen lassen, so vermitteln sie immerhin deutliche Hinweise. Damit wird aber grundsätzlich die Frage nach den Möglichkeiten der Vermittlung von aktuellen geographischen Informationen im Schulunterricht aufgeworfen. Schulbücher enthalten didaktisch aufbereitete Stoffgebiete, veralten aber sehr rasch, während andere Informationsträger (Sachliteratur, Zeitschriften, Statistiken) meist weniger leicht greifbar sind und für die Schule oft lediglich ein Rohmaterial darstellen.

Das Informations- und Dokumentationszentrum für die Geographie der Niederlande

Im Anschluss an eine Konferenz zur Ueberarbeitung geographischer Schulbücher im Jahre 1963 wurde in den Niederlanden die Gründung einer geographischen Informationsstelle angeregt. Eine parlamentarische Anfrage über Fehler in der Darstellung der Niederlande in einem französischen Geographiebuch brachte den Stein ins Rollen. Die geographischen Institute der niederländischen Universitäten, die Königliche Geographische Gesellschaft und mehrere Ministerien arbeiteten bei der Gründung und dem Ausbau des Informations-und Dokumentationszentrums für die Geographie der Niederlande (IDG) mit. Damit entsprachen die Niederlande als erste einer Empfehlung des Europarates.

Das IDG publiziert für ausländische Interessenten - Lehrer, Forscher, Studenten, Schüler, Autoren usw. - geographische Unterlagen über die Niederlande. Diese Schriften sind wissenschaftlich fundiert, aber speziell auf die Bedürfnisse der Schule zugeschnitten.

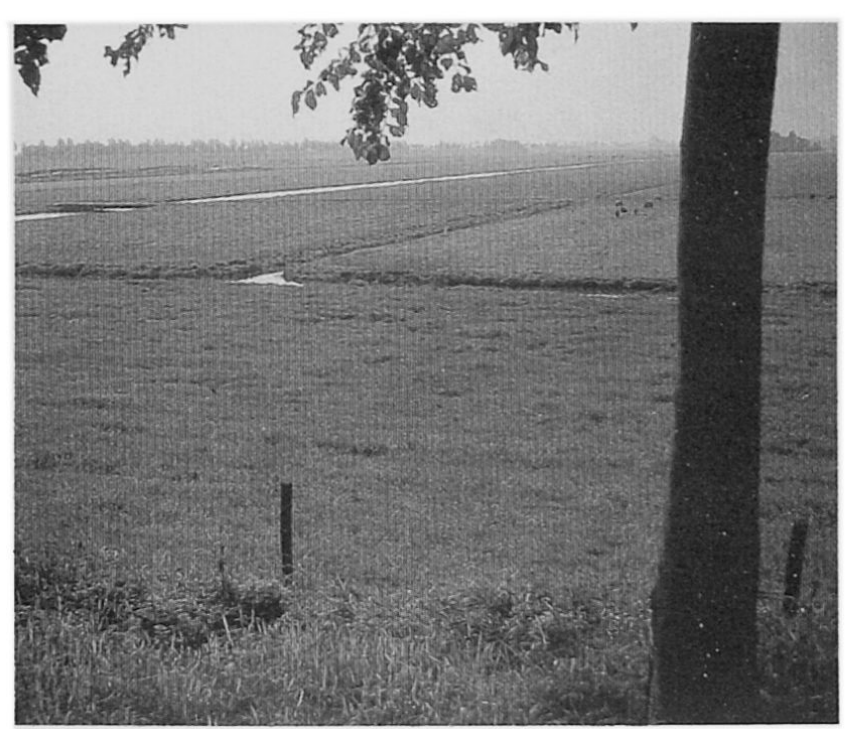

Abb. 3: Das Grüne Herz ist die Lunge der Randstadt: ein landwirtschaftliches Kerngebiet der Grösse eines Schweizerkantons (etwa Zürich oder Freiburg) kann nicht gleichzeitig der Erholung der 6 Millionen Bewohner der umliegenden Städte dienen (jahrhundertalte Polder bei Mijdrecht).

Zu erwähnen sind beispielsweise die "Kleine Geographie der Niederlande", die in 21 Themen die wichtigsten Tatsachen und Probleme der Natur-, Kultur- und Wirtschaftsgeographie des Landes zusammenfasst und der "Bilderatlas der Niederlande", eine reich bebilderte Darstellung von 36 Typlandschaften mit zahlreichen Luftaufnahmen und Karten. Alle Schriften erscheinen in mehreren Sprachen, beispielsweise die "Kleine Geographie der Niederlande" in 13 verschiedenen Fassungen. Sie werden laufend revidiert und erreichen sehr hohe Auflagen.

Diese Publikationen erleichtern dem IDG in entscheidender Art, seinen Auftrag zu erfüllen: allen möglichen Interessenten Auskünfte über die Geographie der Niederlande zu erteilen. Die wichtigsten Publikationen des IDG werden zudem durch die Botschaften und Konsulate der Niederlande in aller Welt verbreitet. Dies ist der Grund, weshalb die diplomatischen Vertretungen der Niederlande neben touristischem Propagandamaterial auch für den Geographieunterricht aller Stufen geeignetes Grundlagenmaterial zur Verfügung stellen können.

Das IDG ist eine kleine, höchst effizient arbeitende Institution. Sie wird durch einen Geographen geführt, dem lediglich eine Teilzeitsekretärin zur Verfügung steht. 1979 wurden 1034 Anfragen beantwortet, seit dem Bestehen der Stelle über 5500 Anfragen. Dies ist nur möglich, weil das IDG einem Hochschulinstitut angegliedert ist (nämlich dem Geographischen Institut der Reichsuniversität Utrecht). Die Stelle wird durch das Unterrichtsministerium und das Ministerium für auswärtige Angelegenheiten finanziell unterstützt. 


\section{Ausblick}

Mit eindrücklicher offenheit stellen die niederländischen Geographen die Probleme ihres kleinen dichtbevölkerten Landes dar. Das IDG spielt dabei nicht die Rolle einer staatlichen Propaganda stelle, sondern stellt einen gemeinnützigen Dienstleistungsbetrieb für einen weltweiten Benützerkreis dar. Es geht schlussendlich um die Verbreitung einer objektiv richtigen, aktuellen Geographie der Niederlande. Das IDG komt insbesondere dem mit Informations- und Dokumentationsproblemen kämpfenden Lehrer aller Stufen entgegen.

Die Niederländer wollen die Ansicht bekämpfen, dass sie nur dank der Deiche und ihrer Holzschuhe trockene Füsse bewahren können und dass sie von Tulpen und Edamer leben.

Vielleicht würde eine geographische Informationsund Dokumentationsstelle auch einem Land gut anstehen, das seinen Klischees - bestehend etwa aus Emmentaler, Schokolade, Uhren, Nummernkonti und Bankgnomen - ernsthaft zu Leibe rücken möchte...
Literatur:

MEIJER, Henk; VERDUIN-MULUER, Henriette, 1979: Towards an authentic geographical image of the Netherlands. An account of the functioning of the Information und documentation centre for the geography of the Netherlands. IDG Utrecht, $31 \mathrm{~s}$.

\section{Adresse des IDG:}

Informations- und Dokumentationszentraum für die Geographie der Niederlande

Rijksuniversiteit Utrecht

Heidelberglaan 2, Postbus 80.115

NL-3508 TC Utrecht/ Niederlande

Publikationen des IDG in deutscher und französischer Sprache

- Kleine Geographie der Niederlande (3.Auflage 1980,44 S.)

- Wandkarte (Massstab 1:325'000)

- Bildatlas der Niederlande (1977, 40 S.)

- Führer durch den Südwesten der Niederlande (2. Auflage 1977, 60 S.)

- Führer Zuidersee/Ijsselmeer (1975, 70 S.

- Führer Randstad Holland (erscheint Ende 1980)

- Verzeichnis deutschsprachiger Literatur über die Geographie der Niederlande

- Verzeichnis französischsprachiger Literatur uber die Geographie der Niederlande

\section{Literaturbesprechungen}

Boje Maassen: Materialien zur Umwelterziehung in allgemeinbildenden Schulen. Band 1 (bis 1974) 143 S, DM 14.--

Div. Autoren: Unterrichtsmaterialien zur Umwelterziehung. Band 2 (bis 1979), 164 S., DM 16.50. Div. Autoren: Umweltunterricht in der Bundesrepublik Deutschland 1980, 257 S., DM 19.80. Helmut Mikelskis: Materialien zum Thema Kernkraftwerke, 127 S., DM 12.50.

Alle: Institut für die Pädagogik der Naturwissenschaften, Kiel; Aulis Verlag Deubner \& Co., Köln.

Das Institut für die Pädagogik der Naturwissenschaften (IPN) ist ein überregionales Zentrum für die interdisziplinäre Forschung, Entwicklung und Lehre im Bereich des naturwissenschaftlichen Unterrichts in allen Bildungsbereichen.

Eine Arbeitsgruppe dieses Instituts befasst sich mit der Veröffentlichung von Informationen unter dem Titel IDK (Information-Dokumentation-Kooperation), v.a. von didaktischem Material.

Die vorliegenden Hefte sind entstanden, da die Bedeutung von verschiedenen Aspekten der Umwelterziehung deutlich zugenommen hat und das Angebot in den traditionellen Schulbüchern der Aktua1 itiit des Themas nicht mehr gerecht wird.
Im Band 1 der 'Materialien zur Umwelterziehung in allgemeinbildenden Schulen" gibt der Verfasser eine Auswahl von geeigneter Literatur zum Bereich Oekologie/Umweltschutz, die bis Sept. 74 in deutscher Sprache vorlag und im Buchhandel erhältlich war. Band 2 bringt eine "annotierte Bibliographie" von Erscheinungen zu diesem Thema im Zeitraum von Sept. 74 bis Sept. 79 . Der Verfasser von 'Materialien zum Thema Kernkraftwerke" legt das Schwergewicht auf bereits erprobte Unterrichtseinheiten zu obigem Thema, $\mathrm{zu}$ welchem er kritische Kommentare liefert (teilweise mit Abbildungen). Ergänzend wird eine $\mathrm{Zu}-$ sammenstellung themenbezogener Zeitschriftenaufsätze, Sachbücher und Filmographie angeboten. Im vierten Band dieser Reihe "Umweltunterricht in der BRD 1980" geht es um eine kritische Bestandesaufnahme nach der Einführung der Umwelterziehung in das allgemeinbildende Schulwesen, im weiteren um Vorschläge zur Verbesserung des Unterrichts, z.B. durch einen koordinierten Lehrplan für die Fächer Biologie und Erdkunde. Als Grundlage wurde das "Umweltgutachten 1978" und eine Arbeitstagung von Biologie- und Geographiedidaktikern in Berlin verwendet.

GH $1 / 81$
Margrit Wegmann, Zürich 